\title{
Criptomoedas: tendência ou realidade? Evidências a partir das demonstrações contábeis das companhias abertas brasileiras ${ }^{1}$
}

\section{Cryptocurrencies: trend or reality? Evidence from the financial statements of Brazilian public companies}

\section{Recebimento: 7/05/2021 - Aceite: 30/06/2021 - Publicação: 01/09/2021 Processo de Avaliação: Double Blind Review}

Juliano Augusto Orsi de Araujo

Professor Titular do Mestrado Profissional em Administração da Universidade Ibirapuera, Brasil

Doutor em Contabilidade pela Universidade de São Paulo, Brasil

juliano.araujo@ibirapuera.edu.br

http://lattes.cnpq.br/8006067851769395

Simone Costa Loia de Araujo

Aluna do Mestrado Profissional em Administração da Universidade Ibirapuera, Brasil

simone@expresscontabil.com.br.

http://orcid.org/0000-0002-1580-295X

\section{RESUMO}

O presente estudo investigou a divulgação das operações e eventuais reservas de criptomoedas nas demonstrações contábeis de 2018 das companhias abertas brasileiras listadas na B3, através da análise de conteúdo das notas explicativas de companhias indexadas no índice IBRX100. A pesquisa ampara-se à Teoria do Disclosure Voluntário. Qualifica-se como pesquisa aplicada e descritiva e com procedimentos de levantamento bibliográfico e busca de dados secundários, através da pesquisa documental, dado que as informações compiladas pelo estudo foram extraídas das notas explicativas das companhias brasileiras listadas na B3. A técnica aplicada foi a análise de conteúdo. A pesquisa identificou o amplo uso do real (BRL) como moeda funcional e o dólar norte americano (USD) como a principal moeda estrangeira. O estudo não encontrou a divulgação do uso de criptomoedas ou ainda a existência de ativos registrados em criptomoedas ou ainda a utilização da cadeia validadora denominada blockchain. Este achado corrobora a teoria do disclosure voluntário e contraria os estudos anteriores que apontam para um uso mais amplo das criptomoedas na atividade empresarial formal. Deste modo, o estudo contribui para a literatura de operações com criptomoedas no Brasil e propõe indagações quanto ao uso e a evidenciação das criptomoedas nas demonstrações contábeis das empresas brasileiras.

\footnotetext{
${ }^{1}$ Este artigo foi apresentado na XX International Finance Conference, na Universidad Catolica de Uruguay, em Montevideo, em Nov/2020.
} 
Palavras-chave: criptomoeda, blockchain, contabilidade, disclosure

\begin{abstract}
This study investigated the disclosure of transactions and any reservations of cryptocurrencies in 2018 financial statements of Brazilian companies listed in B3, through content analysis of the explanatory notes of companies indexed in the index IBRX100. This paper is supported by the Theory of Voluntary Disclosure Information technology aligned to globalization are factors of a significant impact on economic activities worldwide. We used procedures for bibliographic and search of secondary data through documentary research, given that the information compiled by the study were extracted from the explanatory notes of Brazilian companies listed in B3. The technique used was the analysis of content. The research identified the extensive use of real (BRL) as functional currency and the US dollar (USD) as the main foreign currency. The study did not find the disclosure of the use of cryptocurrencies. Or even the existence of assets recorded on cryptocurrencies or even the use of the validator chain called blockchain. This finding corroborates the theory of voluntary disclosure and contradicts the previous studies that point to more extensive use of cryptocurrencies on regular business activity. Thus, the study contributes to the literature on cryptocurrency operations in Brazil. This study proposes questions regarding the use and disclosure of cryptocurrencies in financial statements of Brazilian companies.
\end{abstract}

Keywords: cryptocurrency, blockchain, accounting, disclosure

\title{
1. INTRODUÇÃO
}

A tecnologia em seu avanço contribui para o aumento da velocidade e volume de transações econômico financeiras em uma esfera global. Cernev, Diniz e Jayo (2009) alertam para este fenômeno nas relações bancárias, sobretudo quando se nota maior eficácia nos sistemas de tecnologia da informação, fato que contribui para a ampla divulgação e utilização dos serviços eletrônicos ante aos serviços tradicionais.

Ainda que se perceba e vivencie grandes mudanças tecnológicas, as operações financeiras ainda estão centradas na moeda. Para Guttman (2016), a moeda é a principal forma de representatividade de capital e, segundo o mesmo autor, está sob o controle e emissão de um determinado Estado ou nação, ou órgão por ele reconhecido.

Em meio a este cenário dinâmico e conservador, no ano de 2008, Satoshi Nakamoto apresentou uma proposta de sistema de capital eletrônico para pagamentos cuja tecnologia envolvida é declarada peer-to-peer (ponta a ponta) criptografada, tal que a presença de um 
agente regulador não se faz necessário, pois, para o seu idealizador, o sistema estaria centrado em confiança e em evidências criptográficas (Tiwari, Jana, Das \& Roubaud, 2018).

Essas operações são evidenciadas em uma espécie de livro razão, denominado blockchain e, permite que todos os usuários envolvidos no sistema possam ter acesso às transações ali registradas. Com isto, as operações transparentes entre usuários, são mantidas e fiscalizadas pelos próprios usuários, não fazendo assim a necessidade de um agente regulador ou mesmo fiscalizador.

Urquhart (2018) afirma que a bitcoin é a moeda percursora deste sistema e é modelo para o desenvolvimento das demais que a sucedem. Em função deste pioneirismo, a bitcoin chamou a atenção de investidores e isto resultou em um aumento anormal da criptomoeda. Segundo Dyhrberg et al. (2018), a moeda iniciou suas vendas em agosto de 2010 ao custo de US\$ 0,07 e alcança cerca de US\$ 10.000,00 em setembro de 2019 (Bitvalor, 2019).

Não obstante, Rodríguez e Zúñiga (2018) destacam o uso crescente de criptomoedas no mercado colombiano. Uribe (2019) apresenta em seu estudo, além deste crescimento naquele mercado, o risco para o turismo local em função da não aderência ao uso da criptomoeda, dado o grande número de turistas que optam em pagar suas despesas de viagens ao país sulamericano.

No Brasil, o volume de negociações envolvendo criptomoedas (Exchange), de 2011 a 2019, ultrapassou a casa dos $\mathrm{R} \$ 4$ bilhões, com crescimento acentuado nos últimos meses de abril (R\$ 430 milhões) e maio de 2019 (R \$ 760 milhões). Somente no ano de 2019, a movimentação brasileira alcançou a marca de R \$1,8 bilhões até maio/2019 (Bitvalor, 2019).

Neste sentido, a contabilidade tem que lidar e propor alternativas em relação a movimentação destes ativos, pois, consequentemente, alteram o patrimônio das entidades que são objeto de estudo da Ciência Contábil. No entanto, ainda não há um pronunciamento dos órgãos regulamentadores acerca deste reconhecimento.

Dado o montante apresentado em exchanges no mercado brasileiro e o relevante papel da Ciência Contábil no que tange à evidenciação dos elementos patrimoniais das entidades, o presente estudo se propõe a responder à seguinte questão de pesquisa: como as companhias abertas brasileiras evidenciam as operações e eventuais reservas de criptomoedas em suas demonstrações contábeis? 
Assim, esta pesquisa tem por objetivo investigar acerca da divulgação das operações e eventuais reservas de criptomoedas nas demonstrações contábeis de 2018 das companhias abertas brasileiras listadas na B3. Adicionalmente, identificou as moedas funcionais e estrangeiras adotadas pelas companhias contidas na amostra.

Segundo Araújo (2018), os estudos sobre bitcoins se apresentam crescentes entre 2013 e 2018. Para os autores, os estudos se concentram na investigação comparativa entre índices e propriedades matemáticas, com o uso de técnicas de análises estatísticas como variância e regressão. Os autores afirmam ainda que há escassez de pesquisas sobre a temática em periódicos brasileiros.

Ainda, Baums (2002) afirma que a divulgação das informações pertinentes ao negócio tem por finalidade ser facilitadora no sentido de auxiliar o processo decisório do usuário. Shin (2003) afirma que em momentos de turbulência em mercados, uma das políticas para acalmar os ânimos de investidores é melhorar a divulgação das informações contábeis.

Neste sentido, esta pesquisa vislumbra contribuir com a literatura, preenchendo possíveis lacunas existentes e avançando na produção científica acerca desta temática de disclosure de operações e ativos adquiridos em criptomoedas. Do ponto de vista prático, a pesquisa contribui na construção de uma base de dados que fornece subsídios para estudos futuros que vislumbrem pesquisar acerca do tema.

\section{REFERENCIAL TEÓRICO}

\subsection{Criptomoedas}

As criptomoedas foram criadas por um programador anônimo que adota o nome de Satoshi Nakamoto no ano de 2008. Segundo Ulrich (2014), a moeda virtual funciona inserida em uma rede de pagamentos tal que a operação é realizada diretamente entre duas pessoas, o que o autor denomina de peer-to-peer, par a par, pessoa a pessoa ou simplesmente P2P.

As criptomoedas não são reguladas por nenhuma autoridade competente como o tradicional papel moeda. Segundo Ulrich (2014) é notadamente reconhecido como o sistema de pagamento global totalmente descentralizado. A segurança ocorre pelas chaves criptográficas 
pública e privada envolvidas no processo, além do próprio banco de dados que registra as operações, que pode ser equiparado a um grande livro razão, o blockchain.

Purchio (2017) afirma que as moedas virtuais permitem transações instantâneas e imunes a fraude. Isto pois, a moeda pode ser rastreada desde a sua criação e todas as transações subsequentes, o que não permite que haja a criação de uma criptomoeda falsa. Outro atrativo é o custo da transação: Segundo Purchio (2017), é muito próximo de zero.

Por se tratar de um meio de pagamento presente no mundo virtual e não no meio físico, os órgãos reguladores ainda não estabeleceram normas que regulem as operações. Isto reflete diretamente em questões fiscais e contábeis, dada a alta regulamentação presente em ambas as áreas. Neste sentido, Boff e Ferreira (2016) reforçam a importância da regulamentação acerca das moedas criptografadas vislumbrando maior transparência entre sociedade e estado.

O International Accounting Standards Board (IASB) bem como o Comitê de Pronunciamentos Contábeis (CPC) não possuem opiniões formadas acerca das criptomoedas, seu controle e divulgação das informações produzidas a partir delas. Neste sentido, sem uma regulamentação clara, a divulgação das informações passa a ser voluntária.

\subsection{Disclosure voluntário}

A Teoria do disclosure foi proposta na pesquisa de Verrechia (2001) na qual o autor destaca três categorias de disclosure: i-) divulgação baseada em associação; ii-) divulgação baseada em eficiência e; iii-) divulgação baseada em julgamento.

Para Murcia e Santos (2009) a Teoria do disclosure voluntário oferece o arcabouço conceitual que subsidia as análises das condições em que as companhias divulgam voluntariamente as informações em razão da interação estratégica com agentes externos. Verrechia (2001) afirma que os gestores possuem informações acerca das companhias cuja divulgação não é obrigatória e as usa conforme circunstâncias mais propícias.

O disclosure voluntário apresenta características de divulgação baseada em julgamento, dado que o gestor exerce discricionariedade em relação à decisão de divulgação. Está abarcado pelo arcabouço teórico da Teoria dos Jogos (Dye, 2001). A teoria prediz que a entidade divulga voluntariamente informações favoráveis. Segundo Murcia e Santos (2009), a informação é divulgada voluntariamente apenas se esta gerar algum benefício à empresa. 
Verrechia (2001) afirma que se existem custos para a divulgação de dada informação, isso pode gerar duas reações aos usuários: i-) informações ruins ou negativas ou; ii-) são positivas, porém, com efeito menor do que seu custo de divulgação.

Murcia e Santos (2009) afirmam ainda que a decisão de divulgar ou não determinada informação, está associada ao incentivo que o gestor recebe para se comportar de determinada maneira. Segundo o autor, aceitando-se as premissas de que o objetivo do gestor é o de maximizar o valor da empresa e que existem custos para divulgação de informações, é razoável acreditar que informações que aumentem o valor da empresa sejam divulgadas e aquelas que reduzem ou que possuem caráter negativo, sejam omitidas. Verrechia (2001) admite que os gestores querem que suas empresas sejam avaliadas positivamente.

No entanto, segundo Dye (2001), os gestores nem sempre estão interessados na divulgação destas informações, dada a existência de conflitos de interesses, principalmente na relação de agência. Murcia e Santos (2009) afirmam que os gestores tendem a agir de acordo com seus próprios interesses, divulgando informações boas e favoráveis a ele e retendo as informações ruins.

Ainda quanto à divulgação, o Comitê de Pronunciamentos Contábeis, através do CPC 02 estabelece que as companhias na divulgação de suas demonstrações contábeis devem definir sua moeda funcional e a(s) moeda(s) estrangeiras com as quais a empresa mantem operações econômicas, financeiras ou patrimoniais. Entende-se por moeda funcional aquela do ambiente econômico principal no qual a entidade opera e por moeda estrangeira aquela que é diferente da funcional.

Dada a característica de ausência de órgãos reguladores nas operações com criptomoedas e ainda, por envolver a movimentação de ativos das companhias gestores podem entender que operações com criptomoedas refletem em informações negativas e não divulgam tal informação.

\subsection{Estudos anteriores}

Os estudos sobre criptomoedas são escassos. Araujo, Prado e Araujo (2020) realizaram estudo que levantou a produção bibliográfica sobre a temática contida no Portal de Periódicos da Coordenação de Aperfeiçoamento e de Nível Superior - CAPES - e encontrou 337 artigos publicados. 
Segundo Araujo, Prado e Araujo (2020), os trabalhos se iniciaram em 2013 e consistem, predominantemente, em estudos de natureza qualitativa e são crescentes, sobretudo nos últimos dois anos:

Tabela 1 - Forma de abordagem das publicações

\begin{tabular}{crrrrrr} 
Ano & Qualitativo & \multicolumn{1}{c}{ Quantitativo } & Quali/Quanti & Total & \% \\
\hline $\mathbf{2 0 1 3}$ & 2 & 0 & 0 & 2 & $1 \%$ \\
\hline $\mathbf{2 0 1 4}$ & 14 & 2 & 0 & 16 & $5 \%$ \\
\hline $\mathbf{2 0 1 5}$ & 22 & 2 & 0 & 24 & $7 \%$ \\
\hline $\mathbf{2 0 1 6}$ & 34 & 3 & 0 & 37 & $11 \%$ \\
\hline $\mathbf{2 0 1 7}$ & 73 & 2 & 6 & 81 & $24 \%$ \\
\hline $\mathbf{2 0 1 8}$ & 133 & 23 & 21 & 177 & $53 \%$ \\
\hline Total & 278 & 32 & 27 & 337 & $100 \%$ \\
\hline \% & $82 \%$ & $9 \%$ & $8 \%$ & $100 \%$ &
\end{tabular}

Fonte: Araujo, Prado e Araujo (2020)

Araujo, Prado e Araujo (2020) também indicaram que as publicações foram feitas predominantemente em periódicos internacionais:

Tabela 2 - Publicações por periódicos

\begin{tabular}{lrrr}
\multicolumn{1}{c}{ Periodico } & Publicaçóes & \% \\
\hline Finance Research Letters & 208 & 61,75 \\
\hline Economics Letters & 24 & 7,12 \\
\hline IEEE Access & 15 & 4,45 \\
\hline $\begin{array}{l}\text { Physica A: Statistical } \\
\text { Applications }\end{array}$ & Mechanics and its & 9 & 2,67 \\
\hline Computer & & 8 & 2,37 \\
\hline IEEE Security \& Privacy & 8 & 2,37 \\
\hline Applied Computer Systems & 7 & 2,07 \\
\hline International Review of Financial Analysis & 6 & 1,78 \\
\hline IT Professional & 6 & 1,78 \\
\hline Quarterly Review of Economics and Finance & 6 & 1,78 \\
\hline Bìznes Inform & 5 & 1,48 \\
\hline Future Internet & 5 & 1,48 \\
\hline IEEE Spectrum & 5 & 1,48 \\
\hline Finance Research Letters & 25 & 7,42 \\
\hline Total & 337 & 100,00
\end{tabular}

Fonte: Araujo, Prado e Araujo (2020)

Os aspectos abordados nas pesquisas anteriores se concentraram em, basicamente, 5 classes, assim apresentadas por Araujo, Prado e Araujo (2020): 
a-) análise política e normativa: foco normativo/legal, sua aplicabilidade e viabilidade em conformidade com as normas das instituições financeiras e órgãos fiscalizadores, propondo regulamentações e formulações de políticas monetárias;

b-) comercialização e preço: exame das ligações entre retornos de criptomoedas e atividade de transação, por meio de uma ótica mercadológica, comparando a liquidez comercial com as moedas padrões existentes;

c-) definição conceitual: discussão sobre sua aplicabilidade no mundo real. Principais características por meio de revisões literárias sobre criptomoedas e inovações tecnológicas;

d-) estudo comparativo com índices e propriedades matemáticas: criptografia matemática do código das criptomoedas, com análise de regressão e variância para verificar sua eficiência e possíveis tendências comportamentais;

e-) segurança: consistência das criptomoedas contra-ataques cibernéticos, garantia de retorno e aplicação, com objetivo de evidenciar a confiança de seus usuários.

Nota-se assim a escassez de estudos no Brasil sobre a temática e sobretudo, estudos que investigam a divulgação do uso de criptomoedas por empresas, bem como o disclosure desses ativos nas demonstrações contábeis.

\section{METODOLOGIA}

Este estudo qualifica-se quanto à natureza como uma pesquisa aplicada, com objetivos de pesquisa descritiva e com procedimentos de levantamento bibliográfico e busca de dados secundários, através da pesquisa documental, dado que as informações compiladas pelo estudo foram extraídas das notas explicativas das companhias brasileiras listadas na B3.

A técnica aplicada foi a análise de conteúdo. Foi investigada a presença das palavras "moeda", "criptomoeda", "bitcoin" e "blockchain" nas notas explicativas das companhias contidas na amostra.

Elegeu-se neste momento o exercício de 2018 por ser o último exercício social com a divulgação das informações contábeis completas. Também, para fins de definição da amostra, optou-se em investigar as empresas que compreendem o índice IBRX 100, que consiste no indicador do desempenho médio das cotações dos 100 ativos de maior negociabilidade e representatividade do mercado de ações brasileiro (B3, 2019), em 23.09.2019. 
Ao eleger este índice, são excluídas as companhias em recuperação judicial ou extrajudicial, regime especial de administração temporária, intervenção ou que sejam negociados em qualquer outra situação especial de listagem (B3, 2019). Assim, a amostra final da pesquisa é constituída pelas seguintes empresas:

\begin{tabular}{|c|c|c|c|c|c|}
\hline Ação & $\begin{array}{l}\text { Part. } \\
(\%)\end{array}$ & Ação & $\begin{array}{l}\text { Part. } \\
(\%)\end{array}$ & Ação & $\begin{array}{l}\text { Part. } \\
(\%)\end{array}$ \\
\hline AMBEV S/A & 4,265 & EMBRAER & 0,728 & IOCHP-MAXION & 0,137 \\
\hline AZUL & 0,782 & ENAUTA PART & 0,046 & NATURA & 0,636 \\
\hline B3 & 4,699 & ENERGIAS BR & 0,288 & ODONTOPREV & 0,218 \\
\hline BRASIL & 3,206 & ENERGISA & 0,649 & P.ACUCAR-CBD & 0,657 \\
\hline BRADESCO* & 8,544 & EQUATORIAL & 1,025 & PETROBRAS* & 10,708 \\
\hline BBSEGURIDADE & 1,179 & EZTEC & 0,133 & $\begin{array}{l}\text { PORTO } \\
\text { SEGURO }\end{array}$ & 0,275 \\
\hline MINERVA & 0,066 & FLEURY & 0,388 & QUALICORP & 0,327 \\
\hline BANCO INTER** & 0,451 & GAFISA & 0,022 & RAIADROGASIL & 1,013 \\
\hline BTGP BANCO & 0,565 & GERDAU & 0,688 & RUMO S.A. & 1,282 \\
\hline BRADESPAR & 0,357 & INTERMEDICA & 0,998 & RANDON PART & 0,089 \\
\hline PETROBRAS BR & 1,01 & GERDAU MET & 0,193 & LOCALIZA & 1,305 \\
\hline BRF SA & 1,581 & GOL & 0,227 & $\begin{array}{l}\text { SANTANDER } \\
\text { BR }\end{array}$ & 0,808 \\
\hline BRASKEM & 0,434 & HAPVIDA & 0,547 & SANEPAR & 0,289 \\
\hline BR MALLS PAR & 0,616 & CIA HERING & 0,213 & SABESP & 0,843 \\
\hline BANRISUL & 0,245 & HYPERA & 0,701 & SER EDUCA & 0,063 \\
\hline B2W DIGITAL & 0,463 & IGUATEMI & 0,213 & SLC AGRICOLA & 0,079 \\
\hline CCR SA & 0,97 & IRBBRASIL RE & 1,748 & SMILES & 0,116 \\
\hline CESP & 0,258 & ITAUSA & 3,012 & SUL AMERICA & 0,648 \\
\hline CIELO & 0,475 & ITAUUNIBANCO & 8,337 & SUZANO S.A. & 1,172 \\
\hline CEMIG* & 0,897 & JBS & 2,536 & TAESA & 0,319 \\
\hline CPFL ENERGIA & 0,28 & KLABIN S/A & 0,498 & TENDA & 0,131 \\
\hline COPEL & 0,338 & KROTON & 0,854 & AES TIETE E & 0,182 \\
\hline CARREFOUR BR & 0,433 & LOJAS AMERIC & 0,686 & TIM PART S/A & 0,49 \\
\hline COSAN & 0,426 & LOCAMERICA & 0,194 & TOTVS & 0,385 \\
\hline COPASA & 0,214 & LIGHT S/A & 0,289 & TRAN PAULIST & 0,5 \\
\hline SID NACIONAL & 0,465 & LINX & 0,278 & ULTRAPAR & 1,008 \\
\hline CVC BRASIL & 0,398 & LOJAS RENNER & 1,982 & USIMINAS & 0,211 \\
\hline CYRELA REALT & 0,314 & M.DIASBRANCO & 0,155 & VALE & 7,717 \\
\hline DURATEX & 0,179 & MAGAZ LUIZA & 1,026 & TELEF BRASIL & 1,146 \\
\hline ECORODOVIAS & 0,118 & MARFRIG & 0,215 & VIAVAREJO & 0,43 \\
\hline ENGIE BRASIL & 0,579 & MRV & 0,272 & WEG & 0,914 \\
\hline ELETROBRAS* & 1,031 & MULTIPLAN & 0,393 & YDUQS PART & 0,53 \\
\hline
\end{tabular}


Fonte: B3 (2019)

As empresas marcadas com (*) apresentaram no IBRX100 tanto ações preferenciais quanto ordinárias. Por se tratar da mesma companhia, naturalmente foi excluída a outra ação. A empresa BANCO INTER (**) compôs o índice em 23.09.2019, no entanto, não divulgou informações financeiras em 31.12.2018. Deste modo, a amostra final foi formada por 95 companhias.

\section{ANÁLISE DOS RESULTADOS}

Inicialmente a amostra foi dividida entre empresas financeiras e não financeiras, dada a particularidade de o setor financeiro em relação às suas operações consistirem basicamente de moedas. Das 95 empresas da amostra, 11 empresas são do setor financeiro e 84 companhias não são financeiras:

\section{Gráfico 1 - Amostra}

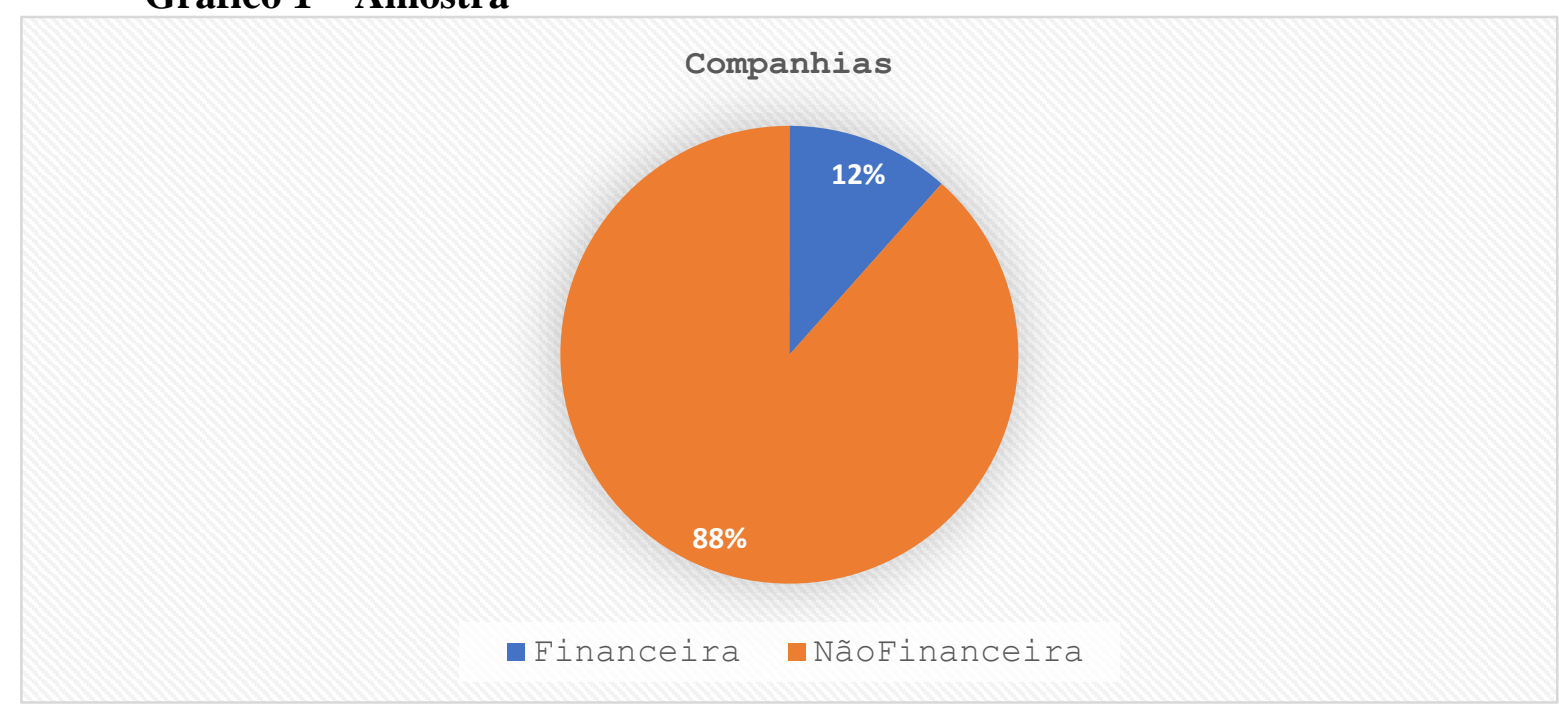

Fonte: dados da pesquisa

Assim, o termo "moeda" foi amplamente mais empregado nas notas explicativas das companhias financeiras se comparadas às não financeiras: 
Tabela 4 - Estatística descritiva

\begin{tabular}{lcccccc} 
& & \multicolumn{5}{c}{ Repetição Moeda } \\
\cline { 3 - 7 } & Obs & Mínimo & Máximo & Média & Mediana & DP \\
\hline Financeira & 11 & 6 & 150 & 64,18 & 33 & 46,38 \\
\hline NãoFinanceira & 84 & 0 & 109 & 26,36 & 22 & 16,04 \\
\hline Fanyyyyyy
\end{tabular}

Fonte: Dados da pesquisa

As medidas centrais para o setor financeiro também são maiores, como observado na tabela 4 para os valores de média e mediana. No entanto, o desvio padrão também se mostrou maior. A moeda funcional foi predominantemente o real (BRL). No entanto, a Embraer foi a única companhia que adotou o dólar norte americano (USD) como moeda funcional. Tal escolha está baseada no fato das operações da empresa brasileira se dar no mercado internacional e, conforme previsto no CPC 02, a escolha é adequada. Observa-se que 3 companhias não declararam sua moeda funcional:

\section{Tabela 5 - Moeda funcional}

\begin{tabular}{ccccc} 
& & \multicolumn{3}{c}{ Moeda Funcional } \\
\cline { 3 - 5 } & Obs & R $\$$ & US\$ & N/D \\
\hline Financeira & 11 & 11 & 0 & 0 \\
\hline NãoFinanceira & 84 & 80 & 1 & 3 \\
\hline Total & 95 & 91 & 1 & 3 \\
\hline
\end{tabular}

Fonte: Dados da pesquisa

Em contrapartida, a adoção do dólar norte americano como moeda estrangeira também foi predominante. Destaque para a Natura que, por decisão da investidora majoritária localizada no Reino Unido, substituiu o euro pela libra esterlina a partir de 01.01.2018. Observa-se que algumas companhias não declararam ou não adotam em função de não manterem operações com o exterior:

Tabela 6 - Moeda estrangeira

\begin{tabular}{lcccc} 
& & \multicolumn{3}{c}{ Operações Internacionais } \\
\cline { 2 - 5 } & Obs & US\$ & $£$ & N/D \\
\hline Financeira & 11 & 10 & 0 & 1 \\
\hline NãoFinanceira & 84 & 75 & 1 & 8 \\
\hline Total & 95 & 85 & 1 & 9 \\
\hline
\end{tabular}

Fonte: Dados da pesquisa 
Por fim, das 95 companhias investigadas na pesquisa, nenhuma delas declarou que mantem algum ativo empregado em criptomoedas ou fez uso de criptomoedas ao longo do exercício social de 2018:

Tabela 7 - Criptomoedas e blockchain Obs Criptomoeda Blockchain

\begin{tabular}{rrrr}
\hline Financeira & 11 & 0 & 0 \\
\hline NãoFinanceira & 84 & 0 & 0 \\
\hline Total & 95 & 0 & 0 \\
\hline
\end{tabular}

Fonte: Dados da pesquisa

Tal fato corrobora os estudos de Dye (2001) e Murcia e Santos (2009) e com o que prediz a teoria de disclosure voluntário (Verrechia, 2001), dado que operações com criptomoedas podem refletir em informações negativas ou ainda, não serem relevantes para usuários e não divulgam tal informação.

\section{CONSIDERAÇÕES FINAIS}

A globalização e a tecnologia da informação são fatores de grande impacto nas atividades econômicas como um todo. Desde 2008 com o surgimento da primeira cadeira de criptomoedas, as transações financeiras também foram atingidas pela tecnologia e a globalização, no entanto, com um aspecto inovador: a ausência de um agente regulador.

Alinhado a isso, a contabilidade tem por finalidade o registro e controle dos eventos econômico-financeiros que impactam no patrimônio das entidades. Dado o crescimento das operações cuja transação se baseia nas criptomoedas, o presente estudo investigou acerca da divulgação das operações e eventuais reservas de criptomoedas nas demonstrações contábeis de 2018 das companhias abertas brasileiras listadas na B3.

Através da análise de conteúdo das notas explicativas do exercício social de 2018 publicadas pelas companhias indexadas no índice IBRX100 em 23.09.2019, a pesquisa identificou o amplo uso do real (BRL) como moeda funcional, exceto para a Embraer, que adota o dólar como moeda funcional em virtude de suas operações se darem amplamente no mercado internacional. 
Assim também o dólar norte americano (USD) se apresentou como a principal moeda estrangeira, excetuando-se a Natura que, por determinação de sua investidora britânica, definiu que a moeda estrangeira é a libra esterlina em substituição ao euro desde 01 de janeiro de 2018.

O estudo não encontrou a divulgação do uso de criptomoedas ou ainda a existência de ativos registrados em criptomoedas ou ainda a utilização da cadeia validadora denominada blockchain.

Este achado corrobora a teoria do disclosure voluntário e contraria os estudos anteriores que apontam para um uso mais amplo das criptomoedas na atividade empresarial formal, como sugerido pelos estudos de Rodríguez e Zúñiga (2018) e Uribe (2019). Este fato pode se dar em função de duas características bastantes distintas entre os estudos: o porte das companhias contidas na amostra - lá empresas menores e de varejo, aqui companhias abertas - e o país de localização das empresas - Colômbia e Brasil.

Assim, o estudo contribui para a literatura ao relatar a evidenciação das (não) operações com criptomoedas no Brasil, sobretudo pelas companhias de capital aberto. Adicionalmente, propõe uma indagação aos futuros pesquisadores acerca de alguns pontos que podem ser esclarecidos: i-) não há divulgação, pois de fato as companhias não mantem operações com criptomoedas; ii-) não há divulgação pelo fato de não existir uma norma que esclareça a maneira mais adequada de divulgação; iii-) não divulga, pois pode ser uma informação negativa ou; iv) não divulga porque não é relevante.

\section{REFERÊNCIAS}

Araújo, V. H. R., Prado, T. A. R., Araujo, J. A. O. (2020). Bitcoin: an analysis of the accounting and finance research published in international journals. In: Annual Meeting American Accounting Association (AAA-2020), 2020, Atlanta - EUA. Annual Meeting American Accounting Association (AAA-2020).

Baums, T. (2002). Changing patterns of corporate disclosure in continental Europe: The example of Germany. European Corporate Governance Institute (EGCI) - Law Working Paper. Social Science Research Network.

Boff, S. O.; \& Ferreira, N. A. (2016) Análise dos benefícios sociais da bitcoin como moeda. Anuário Mexicano de Derecho Internacional, 16, 499-523. 
Cernev, A.; Diniz, E.; \& Jayo, M. (2009). Emergência da quinta onda de inovação bancária. AMCIS 2009 Proceedings. Disponível em <http://aisel.aisnet.org/amcis2009/4>. Acesso em: 25 ago. 2019.

Dye, R. (2001). An evaluation of 'essays on disclosure' and the disclosure literature in accounting. Journal of Accounting and Economics, 32, 181-235.

Dyhberg, A. H.; Foley, S.; \& Svec, J. (2018). How investible is bitcoin? Analyzing the liquidity and transaction costs of bitcoin markets. Economics Letters, 171, 140-143.

Guttmann, R. (2016). A transformação do capital financeiro. Economia e Sociedade, [S.I], 5(2), 51-83.

Murcia, F. D.; \& Santos, A. (2009). Fatores determinantes do nível de disclosure voluntário das companhias abertas no Brasil. Revista de Educação e Pesquisa em Contabilidade, 3(2), 72-95.

Nakamoto, S. (2008). Bitcoin: Peer-to-Peer Electronic Cash System. Disponível em: <https://bitcoin.org/bitcoin.pdf. Acesso 26 ago. 2019.

Purchio, L. (2017). Como usar a moeda digital Bitcoin. Isto é. Publicação de 26 de fevereiro de 2017.

Disponível

em:

$<$ https://istoe.com.br/349307_COMO+USAR+A+MOEDA+DIGITAL+BITCOIN/>. Acesso em 20 ago. 2019.

Rodríguez, N. A. L.; \& Zúñiga, P. A. P. (2018). El Bitcoin como medio de pago en el mercado colombiano. Especialização em Negocios Internacionales. Universitaria Agustiniana, Facultad de Ciencias Económicas y Administrativas, Programa de Negocios Internacionales, Bogotá, Colombia.

Shin, H. (2003). Disclosures and asset return. Econometrica, 71, 105-133.

Tiwari, A. K.; Jana, R.; Das, D.; \& Roubaud, D. (2018). Informational efficiency of bitcoin an extension. Economics Letters, 163, 106-109.

Ulrich, F. (2019). BITCOIN - A moeda na era digital. Disponível

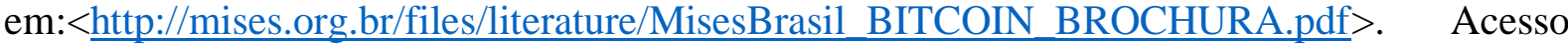
em: 23 de agosto de 2019.

Uribe, J. J. G. (2019). El Bitcoin y el riesgo financiero para el turismo em Colombia. In: International Finance Conference (IFC-2019), 2019, Cordoba - ARG. Anais do International Finance Conference (IFC-2019).

Urquhart, A. (2018). What causes the attention of bitcoin?. Economics Letters, 166, 40-44.

Verrecchia, R. (2001). Essays on disclosure. Journal of Accounting and Economics, 22, 97180. 